Bull. Mater. Sci., Vol. 20, No. 6, September 1997, pp. 823-843. (C) Printed in India.

\title{
Monte Carlo simulation of nucleation and growth of thin films
}

\author{
J GOSWAMI, G ANANTHAKRISHNA ${ }^{\dagger} *$ and S A SHIVASHANKAR \\ Materials Research Centre, Indian Institute of Science, Bangalore 560012 , India \\ ${ }^{\dagger}$ Also at Jawaharlal Nehru Centre for Advanced Scientific Research, Bangalore 560064 , \\ India
}

\begin{abstract}
We study thin film growth using a lattice-gas, solid-on-solid model employing the Monte Carlo technique. The model is applied to chemical vapour deposition (CVD) by including the rate of arrival of the precursor molecules and their dissociation. We include several types of migration energies including the edge migration energy which allows the diffusive movement of the monomer along the interface of the growing film, as well as a migration energy which allows for motion transverse to the interface. Several well-known features of thin film growth are mimicked by this model, including some features of thin copper films grown by CVD. Other features reproduced are-compact clusters, fractal-like clusters, Frank-van der Merwe layer-by-layer growth and Volmer-Weber island growth. This method is applicable to film growth both by CVD and by physical vapour deposition (PVD)
\end{abstract}

Keywords. Monte Carlo simulation; nucleation; growth; thin films.

\section{Introduction}

In general, growth of a thin film is a nonequilibrium process where kinetics and thermodynamics play essential roles in determining the microstructure of the growing film. While kinetics control the tendency of the system to move towards the equilibrium state under a given growth condition, thermodynamics govern the behaviour of the system at equilibrium. The extent to which the system is away from equilibrium depends upon the time periods over which different atomic level kinetic processes are allowed to take place. With the availability of improved surface analysis techniques, such as scanning tunneling microscopy, nucleation and growth of thin films have become a topic of current interest (Gilmer et al 1990; Hwang et al 1991; Mo et al 1991; Michely et al 1993; Roder et al 1993, 1995; Brune et al 1994a, b; Xu and Lu 1994). Recent investigations have uncovered many striking phenomena, pointing to the importance of kinetic processes involved in film growth.

The primary objective of this work is to develop a lattice-gas model which provides a reasonable description of nucleation and growth of thin films. There are several theoretical approaches to the study of thin-film growth. Among the early ones is the rate equation approach, essentially a mean field model which ignores the spatial aspects (Venables et al 1984; Venables 1986). Molecular dynamical methods were applied to the problem of thin-film growth quite early (Gilmer et al 1990; Xu and Lu 1994). It is a powerful approach in which it is possible to

*Author for correspondence 
include atomistic mechanisms. However, in the present context, this method has the same inherent drawback as all molecular dynamic (MD) simulations, namely that the time span of the simulations is small compared to the realistic time span of measurements in experiments. Thus, comparison with experimental results is often difficult. A more popular approach is the Monte Carlo method (Clarke and Vvedensky 1987, 1988; Bartelt and Evans 1994a, b), a mesoscopic approach which permits large-scale computations compared to MD. Further, in the Monte Carlo method, it is easy to mimic atomic level processes such as migration of the growth species, and binding of the desired atoms to the substrate and to one another. In our Monte Carlo model (Goswami et al 1997), we take into account the microscopic processes involved in thin-film growth, such as the deposition rate of atoms on the substrate, their migration leading to the nucleation of islands, capture of atoms by islands, and subsequent coalescence of islands. Using this model, a detailed study has been carried out to understand the competition between the various time scales operating under a given growth condition. Chemical vapour deposition (CVD) has been chosen here as the film growth process simulated, as it allows the treatment to be more general, encompassing the various physical vapour deposition (PVD) methods of film growth, such as MBE. There are inherently three distinct types of time scales in the growth of thin films by CVD, namely the arrival rate of molecules, the dissociation (reaction) rate of the molecules, and the rate of surface kinetics of the growth species released by the dissociation. Of these, the rate of surface kinetics has several components, as considered in detail below. In this work, nucleation and the shape and size distribution of clusters (islands) have been studied under different growth conditions wherein these time scales are varied relative to each other. Investigations have also been carried out to understand the energetics responsible for three-dimensional growth of islands, in contrast with layer-by-layer growth. We establish connections between these simulated growth modes and some experimental results obtained by us. Our simulation results show also that the transition from dendritic to compact island growth involves significant local relaxation via edge diffusion.

\section{Simulation}

Computational and simulation effort is considerably simplified if the model is taken to be a lattice-gas solid-on-solid model (Edwards and Wilkinson 1982; Lai and Sarma 1991; Villain 1991). Here, atoms having a unit volume occupy a unit cell of the same volume on the substrate. In this simulation, the substrate is represented as a square lattice. The flux of deposition species is simulated by a burst of a given number $(N)$ impinging normally on the substrate at specific intervals of time $\left(\Delta t_{f}\right)$. The arrival of the species is assumed to be completely random. The effect of chemical reaction is included in the following way. On arrival at the substrate, the precursor molecules migrate with a certain migration energy which is assumed to be low compared to that of the atomic species constituting the film. The dissociation of the precursor molecules is assumed to take place at a certain rate. On dissociation, the desired atom (monomer) is released and by-products desorb from the substrate immediately after chemical reaction(s). The adsorption of these atomic species takes place instantaneously, as they establish bonds with the substrate. 
Thus, in the case of growth of copper thin films by CVD, for which we present some experimental data, our simulation substrate contains the precursor molecules and copper atoms. In the case of two-dimensional simulation, which we will consider in detail for the sake of illustration, the precursor molecules arriving in later bursts and landing on clusters already formed are considered to be lost. Further, several of the energies introduced below could be taken to be zero, simplifying the computation. Kinetics of the aggregation proceeds in between two successive bursts of molecules. The essential kinetic processes for our purpose are dissociation and surface migration by which adsorbed copper atoms move around and find suitable positions for eventual incorporation into the growing film. Upon collision (i.e. monomers occupying nearest neighbour cells), two monomers form a bond between themselves with a certain probability, as described later. This two-unit cluster acts as a nucleation centre for further growth. In this model, only monomers (for example single copper atoms) are allowed to migrate. Some of the assumptions in our model are routinely used in many simulations (Clarke and Vvedensky 1987, 1988; Kaneko et al 1989; Bartelt and Evans 1994b; Brune et al 1994a; Zhang et al 1994; Ananthakrishna and Noronha 1996).

To study nucleation and growth, different energies considered in this model are as follows:

(i) energy of migration $E_{\mathrm{m}}^{(M)}$ of the precursor molecule before dissociation,

(ii) energy of dissociation $E_{\mathrm{r}}$ of the precursor molecule,

(iii) bond energy between substrate and an adatom, $E_{\mathrm{si}}$,

(iv) bond energy between two nearest-neighbour adatoms, $E_{\text {aa }}$,

(v) migration energy of adatoms on the substrate, $E_{\mathrm{m}}^{(s)}$,

(vi) migration energy of the adatoms on the already-formed film layers, $E_{m}^{(\alpha)}$,

(vii) an additional migration energy $E_{\mathrm{ms}}^{(e)}$ for diffusive motion along the edges (i.e. along the perimeter) of the growing cluster when the cluster is on the substrate,

(viii) a migration energy $E_{\mathrm{ms}}^{(r)}$ for diffusion which is transverse to the motion due to $E_{\mathrm{ms}}^{(e)}$, (i.e. round-the-corner type of diffusion),

(ix) a similar migration energy $E_{\mathrm{m} x}^{(e)}$ for diffusive motion along the perimeter of the growing cluster on the already-grown film, say an island on the first layer,

(x) energy $E_{\text {ma }}^{(r)}$ for diffusion around the corners of an already-grown cluster, which is transverse to the diffusion along the perimeter sites,

(xi) energy of migration from the substrate to the first adatom layer $E_{\mathrm{m}}^{(s, a)}$, and a corresponding energy for migration from the adatom layer to the substrate, $E_{\mathrm{m}}^{(a, s)}$ and

(xii) a similar energy for migration from one adatom layer to another, $E_{\mathrm{m}}^{(i, j)}$, where $i$ and $j$ refer to adjacent adatom layers.

As can be seen, the model incorporates several kinds of energies used in the literature. In particular, the energies introduced in iii-vii have been employed in the literature (Kaneko et al 1989; Gilmer et al 1990; Brune et al 1994a; Ratch et al 1994; Xu and Lu 1994; Zhang et al 1994; Ananthakrishna and Noronha 1996). The physical interpretation of most of them is clear. For instance if $E_{\mathrm{aa}}<E_{\mathrm{sa}}$, it is evident that the formation of the first adatom layer is aided. In v-xii, several types of edge diffusion energies have been introduced and their physical interpretation 
is simple. For instance, a low value of $E_{\mathrm{ms}}^{(e)}$ will allow the atom to find its minimum energy configuration quickly. To the best of our knowledge, it is for the first time that $E_{\mathrm{ms}}^{(r)}$ has been used differently from $E_{\mathrm{ms}}^{(e)}$. (We will often use the term 'interfacial diffusion' both for edge diffusion and for corner diffusion). Other types of edge diffusion energies have similar meaning. The edge diffusion energy for the diffusion of monomers along the interface was specifically introduced in the study of the crossover from diffusion-limited aggregation to dendrites (Ananthakrishna and Noronha 1996). In the present model, many of these energies are held fixed.

In our model, the formation of a nucleus depends on $E_{a \mathrm{a}}$. When two adatoms occupy nearest-neighbour sites, the probability of nucleation is,

$$
P_{\mathrm{v}}=1-e^{\left(-E_{\mathrm{s} /} / k T\right)}
$$

Once nucleation occurs, the cluster of two atoms is assumed to be stable and further dissociation is not allowed. Its subsequent growth into a cluster takes place by capturing monomers. When a monomer is captured by a cluster, it does not necessarily stick immediately to the arrival site, but is allowed to migrate along the interface of the cluster to find a minimum-energy configuration (energies introduced in vii-x). This model considers infinite potential boundary walls, i.e. adatoms get reflected back when they reach the boundaries. Even though $E_{\mathrm{sa}}$ is introduced in this model, in the case of two-dimensional simulation, it sets only a scale in determining the energy $E_{\text {aa }}$ and can only manifest if desorption is considered. However, desorption is ignored because of the low-growth temperature $\left(280-450^{\circ} \mathrm{C}\right)$ employed for the CVD of $\mathrm{Cu}$ films discussed below.

Monte Carlo simulation is implemented, using the transition rate from an initial to a final state, consistent with the principle of detailed balance, namely

$$
W_{\mathrm{i} \rightarrow \mathrm{f}}=R \frac{v}{2} e^{-} E^{(v) / k T} \cdot e^{-\Delta E_{i \rightarrow 1} / k T}
$$

where $E^{(v)}$ refers to the appropriate energy of migration and $\Delta E_{\mathrm{i} \rightarrow \mathrm{f}}$ is the change of energy between the two states. $R$ refers to the rate of dissociation of the precursor molecules taken as

$$
R=R_{0} \exp \left(-E_{r} / k T\right)
$$

For further considerations, $v=1$ and $R_{0}=1$ are used.

The time interval $\Delta t_{\mathrm{b}}$ between two successive bursts of atoms can be subdivided into an integral number $(b)$ of smaller units of time $\Delta t_{\mathrm{m}}$ such that

$$
\Delta t_{\mathrm{b}}=b \cdot \Delta t_{\mathrm{m}}
$$

In any time interval $\Delta t_{\mathrm{m}}$, each adatom on the substrate is tested (on an average) once for migration and in the time interval $\Delta t_{\mathrm{b}}$, such test is executed $b$ times. Thus, the Monte Carlo unit (MCU) of time is $\Delta t_{\mathrm{m}}$. As at any instant of time, the Monte Carlo trial is made only on one atom, $\mathrm{i} \rightarrow \mathrm{f}$ specifies the change in the state of the entire system as that due to a particular adatom which is tested for migration.

Computational time needed is the major obstacle to carrying out large-scale 
simulations that approximate 'real' experiments. For instance, a typical simulation with substrate size $50 \times 50$ and $\Delta t_{\mathrm{b}}=1000$, carried out on an IBM 580 computer, consumed a CPU time of $90 \mathrm{~min}$. Therefore, the size of a typical simulation was restricted to $50 \times 50$ units, even though some specific calculations were carried out on a $200 \times 200$ substrate. As the various diffusion energies and other kinetic data relating to film growth are generally not available, growth parameters have been chosen over a wide range of values to simulate film growth under a variety of conditions. Absolute values of the parameters do not have any serious meaning, but their relative values are important for the study of their effects on morphological features and to understand growth mechanisms. All migration energies are scaled in units of $k T$. The time interval between two successive bursts of atoms, $\Delta t_{\mathrm{b}}$, was again chosen based on available CPU time. Although a variety of growth conditions representing combinations of incident vapour flux $(N)$, different adatom-adatom binding energies $\left(E_{\mathrm{a} a}\right)$ and different migration energies have been employed, we attempt a direct correlation between simulation results and experimental data for CVD-grown copper films only for variations of $N$ and of the surface migration energies $E_{\mathrm{n}}^{(s)}$, and the interfacial energies.

\section{Results and discussion}

It is obvious that in the case of CVD film growth, $E_{\mathrm{r}}$ is a crucial parameter. We refer the reader to Goswami et al (1997) for a detailed investigation of the CVD case. It is clear that, when $E_{\mathrm{r}}=0$, the dissociation of the molecules is instantaneous. This assumption is applicable for some CVD precursors, like bis(dipivaloylmethanato) $\mathrm{Cu}(\mathrm{II})$ or $\mathrm{Cu}(\mathrm{dpm})_{2}$, as shown earlier (Goswami et al 1997). Thus, the monomers in this simulation correspond to the atoms comprising the film. Since precursor dissociation is assumed to be instantaneous, only two basic time scales, one corresponding to the arrival rate of adatoms and the other relating to the surface energetics, are important. The influence of the variation of these two time scales on film growth has been investigated here. Specifically, the relative magnitude of the adatom-adatom binding energy compared to the substrate-adatom binding energy was varied to study its effect on film growth. It is to be noted that the unit of time (MCU) used here is the Monte Carlo unit of time. All migration energies are scaled in units of $k T$. The time interval between two successive bursts of atoms $\Delta t_{\mathrm{b}}$ was again chosen on the basis of available CPU time. $\left(\Delta t_{\mathrm{b}}=1000 \mathrm{MCU}\right)$. In the following section, we shall refer to $E_{\mathrm{ms}}^{(r)}, E_{\mathrm{ms}}^{(r)}$ as $E_{\mathrm{m}}^{(c)}$ and $E_{\mathrm{m}}^{(r)}$ except when the discussion refers to three-dimensional growth. We shall also use the notation $E_{\mathrm{m}}^{(\ell / r)}$ while referring to $E_{\mathrm{m}}^{(\ell)}$ and/or $E_{\mathrm{m}}^{(r)}$.

\subsection{Sub-monolayer growth}

In the simulation of sub-monolayer film growth at low coverage, deposition of atoms on top of islands is ignored. As adatoms migrate only on the substrate surface, the substrate-adatom binding energy does not play any role. Growth was characterized by island density, size distribution of islands, and the snapshot of growth configuration at a given coverage. Simulations were done to study the nucleation and growth process as a function of time under a given deposition 
condition (keeping the arrival rate and all energies constant). Effects of the arrival rate of atoms and of different surface diffusion rates on the early stages of film growth were investigated. Morphological evolution as a function of time was also studied under different growth conditions. Simulation results thus obtained have been used to interpret the observed morphologies of copper films grown by CVD in our laboratory.

3.1a Nucleation: Three basic processes can be identified in the early stages of film growth. At low coverage, the atoms deposited hop on the substrates as 'random walkers' until they form di-atom clusters (which are the critical nuclei in the present case) due to the near neighbour adatom-adatom interaction. These nuclei subsequently grow by capturing additional atoms, thus increasing surface coverage. At high coverage, two or more clusters merge into one large cluster, and coalescence becomes important. At low coverage when coalescence is unimportant, the following set of simulations were carried out to study only the nucleation process. A single burst of 1000 atoms was considered to impinge on a substrate of $200 \times 200$ sites. Observations were made at different times up to $2000 \mathrm{MCU}$. Growth parameters used for the simulations were: $N=1000, E_{\mathrm{aa}}=1 \cdot 9, E_{\mathrm{m}}^{(s)}=0 \cdot 19, E_{\mathrm{m}}^{(e)}=0.0019$, and $E_{\mathrm{m}}^{(r)}=0.0019$. Snapshots of the substrate taken at different times are shown in figure 1 , where monomers are not shown. It is interesting to note the similarities between these snap shots and the STM micrographs of Brune et al $(1994 \mathrm{a}, \mathrm{b})$ for low coverages of $\mathrm{Ag}$ on $\mathrm{Pt}$.

The size distribution of atomic clusters as a function of time is shown in figure 2. Quite early $(\sim 10 \mathrm{MCU})$, one sees a small fraction of the monomers go into the nucleation of di-atom clusters. Subsecuently. the nucleation density increases sharply
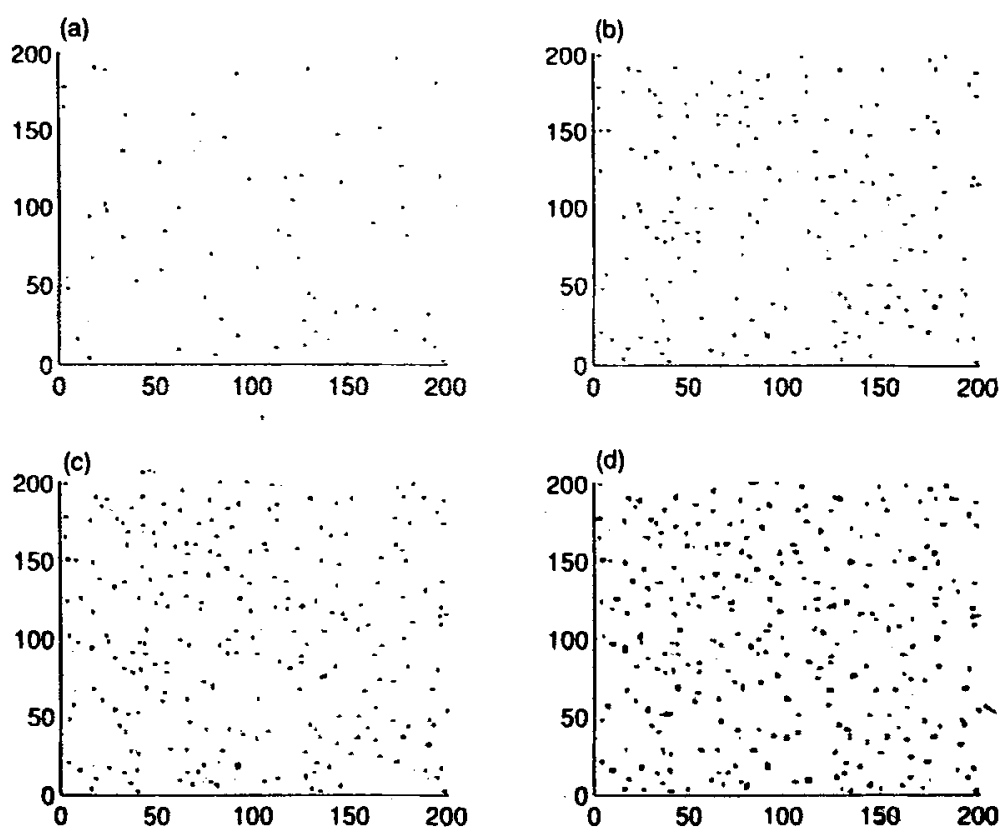

Figure 1. Sequence of growth configurations with increasing time: (a) $10 \mathrm{MCU}$, (b) $50 \mathrm{MCU}$, (c) $100 \mathrm{MCU}$ and (d) $200 \mathrm{MCU}$. Monomers are not shown. 
up to $100 \mathrm{MCU}$, by which time nearly saturation density of nuclei $\sim 88 \%$ is seen. Larger clusters are seen only after this time. As the growth process proceeds, the total number of adatoms captured by the nuclei increases, overtaking the rate of formation of nuclei, and leading to the decrease of nucleation density. This result can be understood on the basis of two length scales involved in the growth process. The first one is the mean distance $l_{N}$ between the monomers at any given instant of time, in particular at the beginning of the nucleation stage. (Note that the monomers arrive and move randomly). The other length scale is the diffusion length, $l_{\mathrm{D}}$, of the monomers. (Here, we are referring to the surface diffusion length.) The latter does not change with time, but the former is changing rapidly due to the constant rate of arrival of the monomers. This means that, initially, $l_{N}^{2}$ increases linearly. As the collision of monomers is controlled by $l_{D}$, which is constant, the nucleation density increases linearly at the initial stages. As the nucleation density increases, the mean distance between the nuclei controls the subsequent growth, since the probability of collision of a monomer with the already formed nuclei (or bigger clusters) increases. At this stage of growth, clusters grow
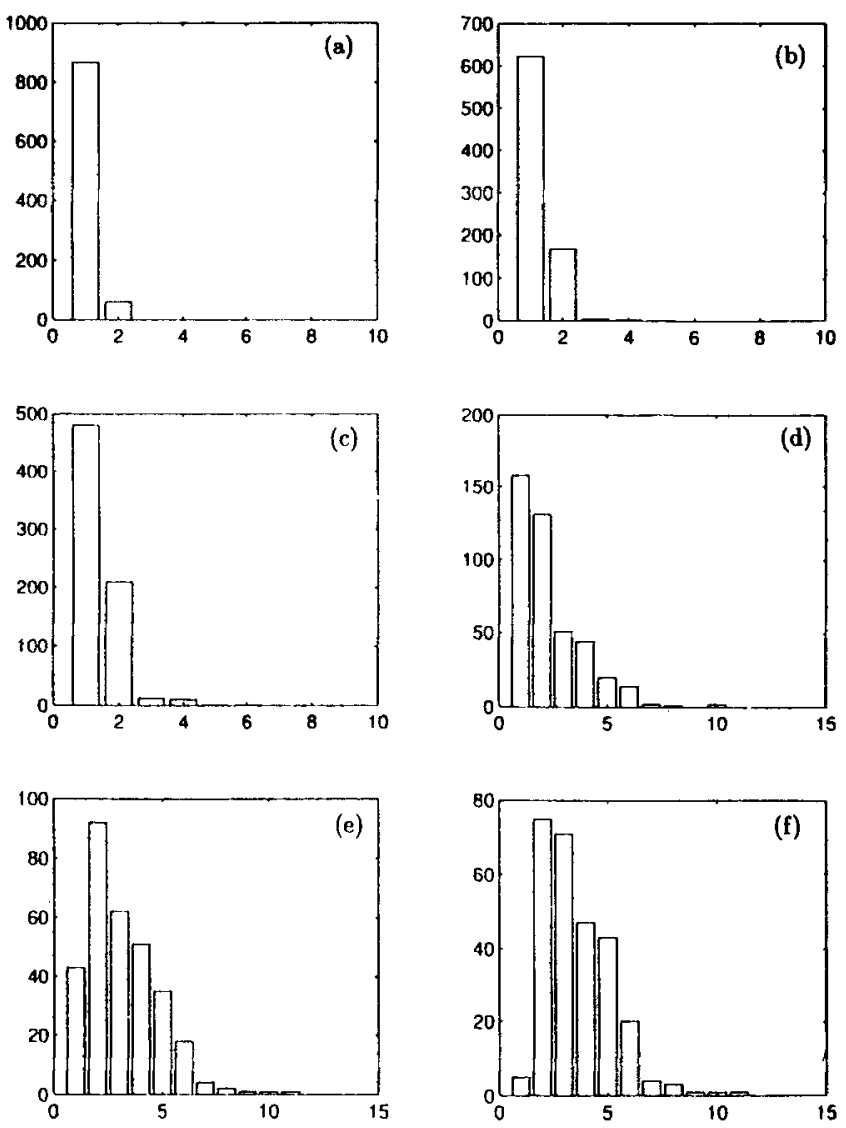

Figure 2. Size distribution of cluscls as a function of time: (a) $10 \mathrm{MCU}$, (b) $50 \mathrm{MCU}$, (c) $100 \mathrm{MCU}$, (d) $500 \mathrm{MCU}$, (e) $1000 \mathrm{MCU}$ and (f) $2000 \mathrm{MCU}$. 
by depleting monomers from the substrate. Further growth of the clusters proceeds by further absorption of the monomers at a slow pace. In the present case, the nucleation density levels off slowly and becomes saturated approximately at $t=200 \mathrm{MCU}$. Thus, the density of di-atom clusters decreases with time as shown in figure 2. As time progresses, clusters with higher number of monomers such as three, four and up to ten, are seen by $500 \mathrm{MCU}$. Thus the growth process could be broadly divided into two stages: one corresponding to a rapid nucleation stage where formation of only two-atom clusters occurs, and the other corresponding to a prolonged continuation of cluster growth. A plot of the nucleation density as a function of time is shown in figure 3.

3.1b Effects of arrival rate: To investigate the effect of the arrival rate of atoms (growth species) on cluster density and on the size distribution of clusters, the number $(N)$ of incident atoms per burst was varied. Growth parameters were chosen as $E_{\mathrm{aa}}=1.9, E_{\mathrm{tn}}^{(s)}=0.19, E_{\mathrm{m}}^{(c)}=0.019$, and $E_{\mathrm{m}}^{(r)}=0.019$. The following four cases were considered for simulation: (i) $N=200$, (ii) $N=100$, (iii) $N=50$, and (iv) $N=10$. The total number of atoms involved in the growth was kept approximately equal to 200 , corresponding to a fixed sub-monolayer coverage of nearly 0.08 monolayer (ML) in each case. The growth configurations for different values of $N$ are shown in figures $4 a-d$.

According to heterogeneous nucleation theory, reduction in deposition rate is expected to lower the nucleation density (Venables et al 1984) which is what is seen in our simulation. This can be seen again by considering the two length scales $l_{N}$ and $l_{\mathrm{D}}$ in the problem stated in the previous section. Since $l_{N} \sim N^{-1 / 2}$, for $l_{\mathrm{D}}$ constant, at the initial stages of film growth, higher arrival rate implies that an adatom on an average travels a shorter distance before it meets another atom to form a nucleation centre. This leads to increased cluster density. Figure 5 shows the number of nucleation centres as a function of time. For $N=200$, nucleation density attains a saturation value much faster than for $N=10$. In the latter case,

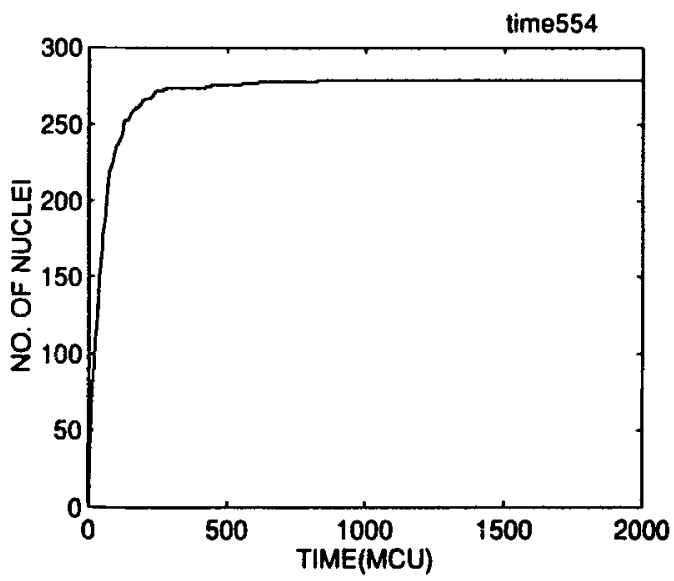

Figure 3. Tobal number of nuclei on the substrate as a function of time. 
nucleation continues along with the growth of clusters. Further, in figure $4 d$, when the arrival rate is very low $(N=10), l_{N}$ is small, implying smaller collision probability of the monomers. Therefore the probability of capture of an adatom by the already formed nuclei is greater. This probability itself increases as the cluster grow, resulting in the formation of larger clusters of different sizes at low density. Concomitantly, there will be a certain amount of nucleation also.

These simulation results compare well with the experimental data obtained in the case of CVD of copper, where a high molar flow rate of the metalorganic precursor, bis(dipivaloylmethanato) $\mathrm{Cu}(\mathrm{II})$ or $\mathrm{Cu}(\mathrm{dpm})_{2}$, resulted in dense morphology. At a lower molar flow rate, a porous microstructure with a wide variation of grain sizes is formed (figure 6).
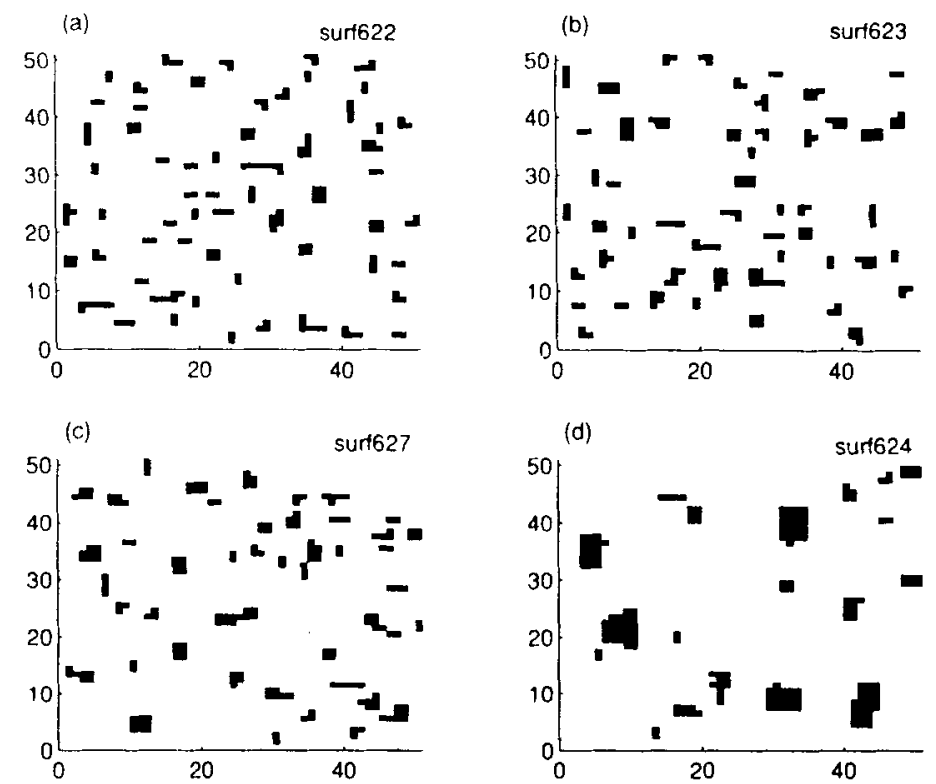

Figure 4. Growth configurations for different values of $N$ : (a) 200 , (b) 100 , (c) 50 and (d) 10 .
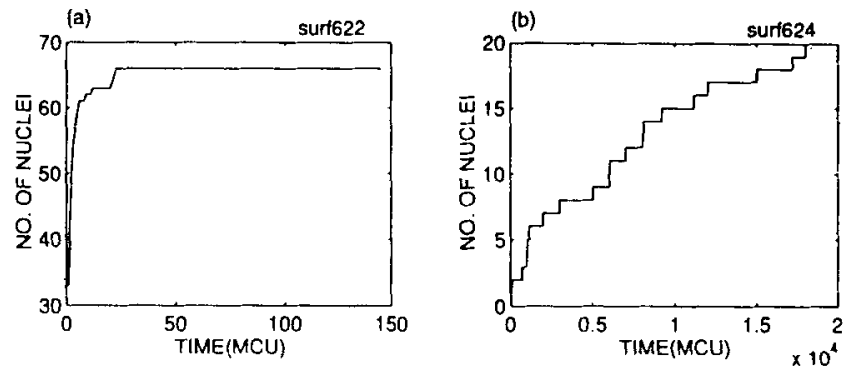

Figure 5. Total number of nuclei on the substrate as a function of time, (a) $N=200$ and (b) $N=10$. 
3.1c Effects of surface diffusion: Kinetic relaxation governed by different surface diffusion rates can force adatoms to move towards a minimum energy configuration, and hence film growth may take place under near-equilibrium conditions. To understand the effects of surface diffusion rates on cluster shape and distribution, the following set of simulations were carried out. Migration energies were varied over a wide range of values keeping $E_{m}^{(s)}: E_{m}^{(e)}: E_{m}^{(r)}$ constant at $19: 1: 1$ in all simulations. $E_{\mathrm{m}}^{(\ell)}$ and $E_{\mathrm{m}}^{(f)}$ were kept much lower than $E_{\mathrm{m}}^{(\mathrm{s})}$, so that any adatom captured by a cluster moves rapidly along the perimeter sites to find the minimum energy configuration. A parameter $\varepsilon$ was introduced such that $E_{\mathrm{m}}^{(s)}=\varepsilon$,
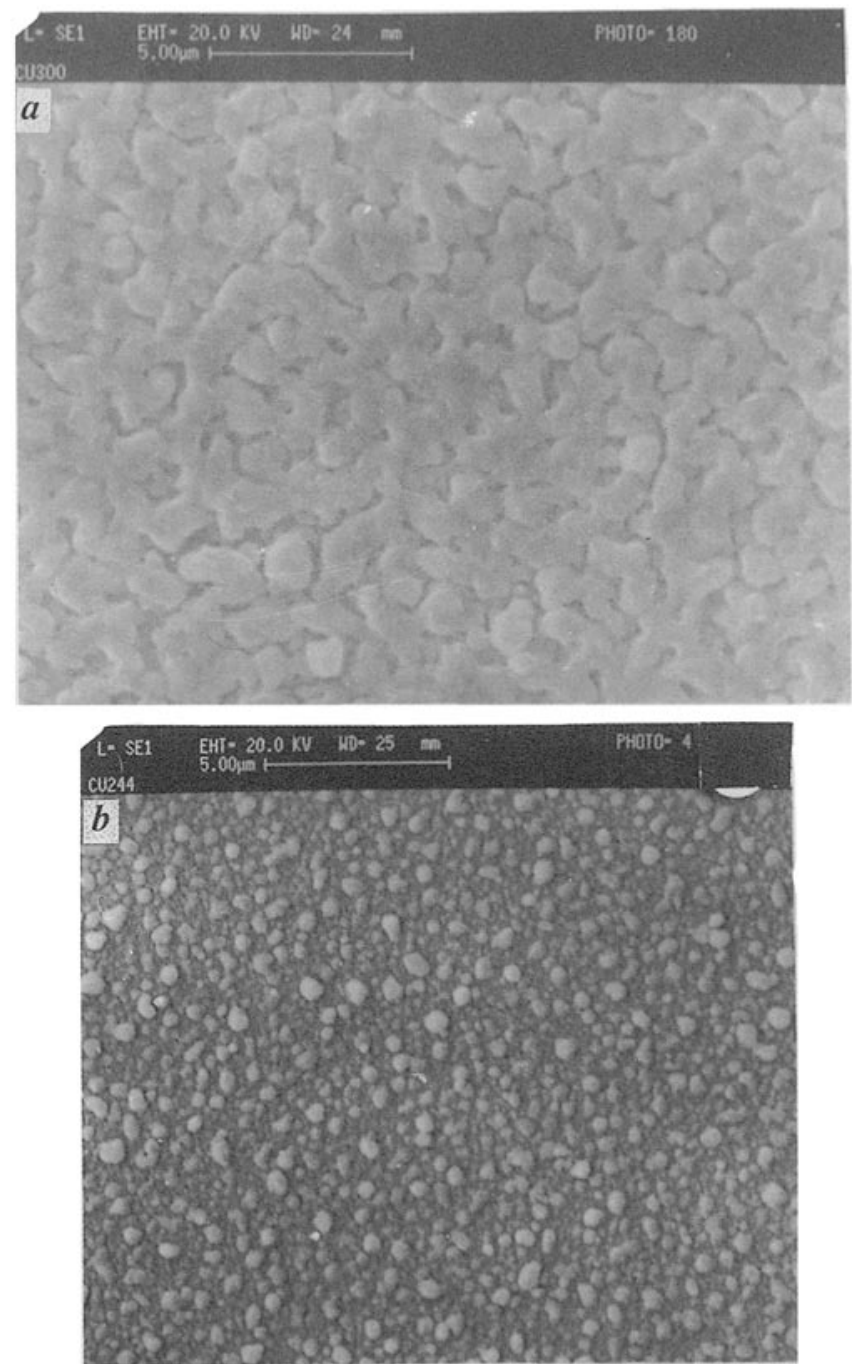

Figure 6. SEM micrographs of copper films grown from $\mathrm{Cu}(\mathrm{dpm})_{2}$ at different total gas flow rates: (a) $200 \mathrm{sccm}$ and (b) $50 \mathrm{sccm}$. Pressure, $P=10$ Torr. Corresponding thicknesses were, respectively, $3200 \AA$ and $750 \AA$. 
$E_{\mathrm{m}}^{(e)}=0.19 \times \varepsilon$, and $E_{\mathrm{ll}}^{(r)}=0.19 \times \varepsilon . \varepsilon$ was varied from a very high value of 0.3 to a very low value of 0.001 . A high value of $\varepsilon$ implies low surface mobility. Other growth parameters set for the experiments were $N=10$ and $E_{\mathrm{ad}}=1.9$. A total number of 100 bursts of atoms was made for each experiment, keeping coverage constant at $0.32 \mathrm{ML}$. Figure 7 shows the growth configuration for different values of $\varepsilon$.

These results illustrate that a higher migration energy $\varepsilon$ leads to higher nucleation density for a fixed arrival rate, and to the formation of smaller size clusters and dense morphology. Under the given growth conditions, the total number of nucleation centres at $\varepsilon=0.3$ is 172 , compared to 42 at $\varepsilon=0.001$. One also notices that when $\varepsilon$ drops below $0 \cdot(01$, growth morphology appears to approach well-separated larger clusters with sharp edges than at lower value of $\varepsilon$. At a very low value of $\varepsilon$ $(=0.001)$, individual clusters grow under near-equilibrium conditions and tend to be square-shaped, reflecting the symmetry of the substrate. It should be noted that when $E_{\mathrm{n}}^{(u / r)}$ is very small, the adatoms captured by the clusters find the minimum energy configuration very quickly. These results are easy to understand in terms of $l_{\mathrm{D}}$ which determines the nucleation density. If the rate of deposition is low, and $l_{\mathrm{D}}$ is small (at high $\varepsilon$ ), few nuclei are formed until the number of monomers increases to such a value that $l_{N} \sim l_{\mathrm{D}}$, whereupon collisions occur between monomers. The cluster density is largely determined by the initial nucleation density formed at this stage. For a constant rate of deposition, $l_{D}$ is larger for lower $\varepsilon$, and hence the nucleation density is lower.

It is clear from the above considerations that when $E_{m}^{(e / r)}$ is high, the captured monomers cannot move quickly and hence there will be a larger number of uncompensated bonds. (This will be treated in some detail.) Thus, a large value
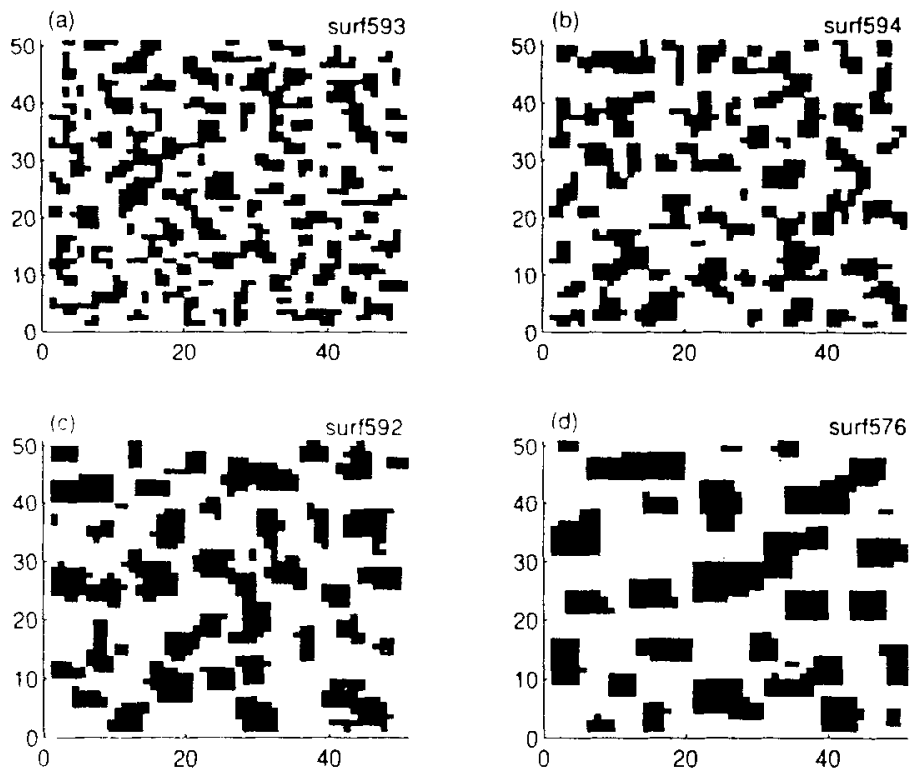

Figure 7. Growth configurations for different values of $\varepsilon$ : (a) $0 \cdot 3$, (b) 0.25 , (c) 0.2 and (d) 0.001 
of the total perimeter $p$ corresponds to a higher energy configuration of the system. Hence, the total $p$ of the system (i.e. over all the clusters) can be taken to represent the closeness of the system to equilibrium. Figure 8 shows the plot of $p$ as a function of $1 / \varepsilon$.

A sharp reduction in $p$ was observed as $\varepsilon$ was reduced from 0.3 to $0.01 ; p$ decreased slowly with further decrease in $\varepsilon$. This is due to the exponential dependence of migration probability on $\varepsilon$. The results illustrate that a smaller $\varepsilon$ brings the system closer to equilibrium. In experiments on the CVD of copper from $\mathrm{Cu}(\mathrm{dpm})_{2}$, described elsewhere (Goswami 1995), a dense and well-connected microstructure was formed at a low substrate temperature of $350^{\circ} \mathrm{C}$, while larger grains forming a porous microstructure were observed at a high substrate temperature of $450^{\circ} \mathrm{C}$ (figure 9). It should be noted that a higher substrate temperature implies a higher surface mobility (i.e. $\varepsilon$ is small) of the adsorbed growth species. Simulation results thus describe correctly the qualitative features of our experimental data.

3.1d Evolution of surface morphology: In this section we investigate the effect of arrival rate on the microstructure at two distinct surface diffusion rates. As discussed earlier, a higher arrival rate at a fixed surface diffusion energy gives higher nucleation density and greater connectivity at a given coverage. Under these conditions, if the surface diffusion is set to be 'fast', the clusters are closer to their equilibrium configuration when the arrival rate is low than if it is high. When surface diffusion is comparatively slow, the nucleation density and cluster connectivity is higher at a given coverage than it is when diffusion is faster (figure 10). The formation of dense, fine grained copper films which are continuous at a very low thickness $(10 \mathrm{~nm})$ from the pyrolysis of $\mathrm{Cu}(\text { tbaoac) })_{2}$ is illustrative of such growth (Goswami et al 1996, 1997).

3.1e Effects of $E_{\mathrm{m}}^{(e)}$ and $E_{\mathrm{m}}^{(r)}$ : Growth of a cluster towards the minimum energy configuration depends on two competing parameters, one corresponding to the capturing rate of an adatom by an existing cluster, and the other one corresponding to the diffusive relaxation of an adatom along the interface of the cluster. The

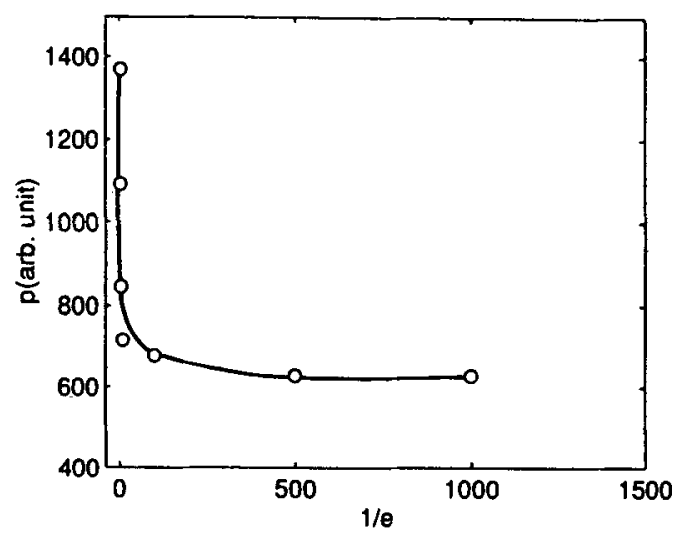

Figure 8. Plot of $p$ as a function of the perimeter $l / \varepsilon$. 
following sets of simulations were done to study the effects of diffusive relaxations on cluster growth. In this case, the capture rate of the adatoms was kept approximately the same by choosing the barrier for surface migration energy $E_{m}^{(s)}(0 \cdot 19)$ identical for the entire set of simulations. For the first set of simulations, $E_{\mathrm{m}}^{(e)}$ and $E_{\mathrm{m}}^{(r)}$ were set equal, and a new parameter $u$ was introduced such that $E_{\mathrm{m}}^{(e)}=E_{\mathrm{m}}^{(r)}=u \cdot E_{\mathrm{m}}^{(s)} \cdot u$ was varied over a wide range from 0.001 to 50 . Other growth parameters were kept as $E_{\mathrm{a} a \mathrm{a}}=1.9$ and $N=2$. The total number of bursts made on the substrate was 250. Figure 11 shows growth of clusters for four different values of $u$ at the same coverage of $0.18 \mathrm{ML}$.

At a high value of $u$ corresponding to 50 (figure $11 \mathrm{a}$ ), the clusters are ramified and resemble the fractal structures produced in two-dimensional DLA models (Witten and Sander 1981; Brune et al 1994a; Ananthakrishna and Noronha 1996). This is because when both the migration energies $E_{\mathrm{m}}^{(\ell)}$ and $E_{\mathrm{m}}^{(r)}$ are high, an adatom has very little tendency to diffuse along the perimeter of the aggregate, resulting in DLA-like structure. Using STM, Hwang and co-workers have experimentally observed diffusion-limited, fractal-type growth of clusters when Au was deposited on $\mathrm{Ru}(0001)$ substrate under sub-monolayer coverage (Hwang et al 1991). With decreasing $u$, diffusion along the perimeter sites begins to play an important role and the shapes of the clusters approach a more compact form (figure 11b). At a much lower value of $u$, corresponding to 0.01 (figure $11 \mathrm{~d}$ ), the diffusive relaxation is very rapid. This brings the system closer to equilibrium, and individual clusters tend to reach their minimum energy configuration (square shape) (Brune et al 1994a; Ananthakrishna and Noronha 1996).

In figure 12, total perimeter $p$ (estimated by measuring the perimeters of the individual clusters) of the system is plotted against $1 / u$. It is clearly seen that energy of the system decreases rapidly with decreasing $u$ up to $0 \cdot 1$. At lower values of $u$, the system energy approaches the minimum value slowly. The variation of $p$ as a function of $u$ can be explained on the basis that diffusive relaxation (corresponding to edge and corner diffusion rate) depends exponentially on $u$.

In the second set of simulations, relative magnitudes of $E_{\mathrm{m}}^{(e)}$ and $E_{\mathrm{m}}^{(r)}$ were varied to study its influence on growth morphology. The following possibilities were considered: $E_{\mathrm{m}}^{(e)}=E_{\mathrm{m}}^{(r)}, E_{\mathrm{nt}}^{(e)}<E_{\mathrm{n}}^{(r)}$, and $E_{\mathrm{m}}^{(e)}>E_{\mathrm{m}}^{(r)}$. While $E_{\mathrm{m}}^{(e)}$ governs the diffusive motion of an adatom parallel to the flat interface of a cluster, $E_{\mathrm{m}}^{(r)}$ controls the local transverse motion around a sharp corner. Growth parameters used for the simulations were: $N=2, E_{\mathrm{aa}}=1.9, E_{\mathrm{m}}^{(.)}=0 \cdot 19$. A total number of 250 bursts of atoms was made to get a surface coverage of $\sim 0.18 \mathrm{ML}$. The following parameters were introduced for this study: $E_{\mathrm{m}}^{(e)}=y \cdot E_{\mathrm{m}}^{(s)}$, and $E_{\mathrm{m}}^{(r)}=z \cdot E_{\mathrm{m}}^{(s)}$. First, $E_{\mathrm{m}}^{(e)}$ and $E_{\mathrm{m}}^{(r)}$ were kept equal such that $y=z=50$. The resulting fractal type structure is illustrated in figure $11 \mathrm{a}$. When $E_{\mathrm{m}}^{(r)}>E_{\mathrm{m}}^{(r)}$, edge diffusion is more dominant than corner diffusion. In this case, growth of individual clusters occurs with wider branches, as presented in figure 13a, where $y=0.01$ and $z=50$. This is because, as $E_{\mathrm{m}}^{(r)}$ is very high, diffusion around sharp corners is unfavourable and the adatoms have a tendency to aggregate at kink sites (figure 13a), leading to thicker arms (more like dendrites) (compare figure 11a). When $E_{m}^{(r)}$ is lower, individual clusters become more compact as shown in figure $13 \mathrm{~b}$, where $y=50$ and $z=0.01$. Similar results have been obtained by Ananthakrishna and Noronha (1996) recently in the context of the study of the crossover from diffusion limited aggregate to dendrite structure. 


\subsection{Three dimensional growth}

Film growth takes place in one of three modes, (i) Frank-van der Merwe or layer-by-layer growth mode (FM), (ii) Volmer-Weber (VW) or island growth mode, and (iii) Stranski-Krastanov (SK) or layer-plus-island growth mode (Venables et al 1984). The present model in its simple form clearly simulates the first two growth modes as described below. Simulations were carried out to understand the influence of different binding energies, such as the adatom-adatom binding energy $E_{\text {aid }}$, and the adatom-substrate binding energy $E_{\mathrm{s} a}$ in determining the film growth mode. To investigate the growth modes, the following three cases were considered: $E_{\mathrm{aa}}<E_{\mathrm{su}}$, $E_{\mathrm{ua}}=E_{\mathrm{sal}}$ and $E_{\mathrm{aa}}>E_{\mathrm{sa}}$. Growth parameters set for these simulations were: $E_{\mathrm{m}}^{(s)}=0 \cdot 19$,
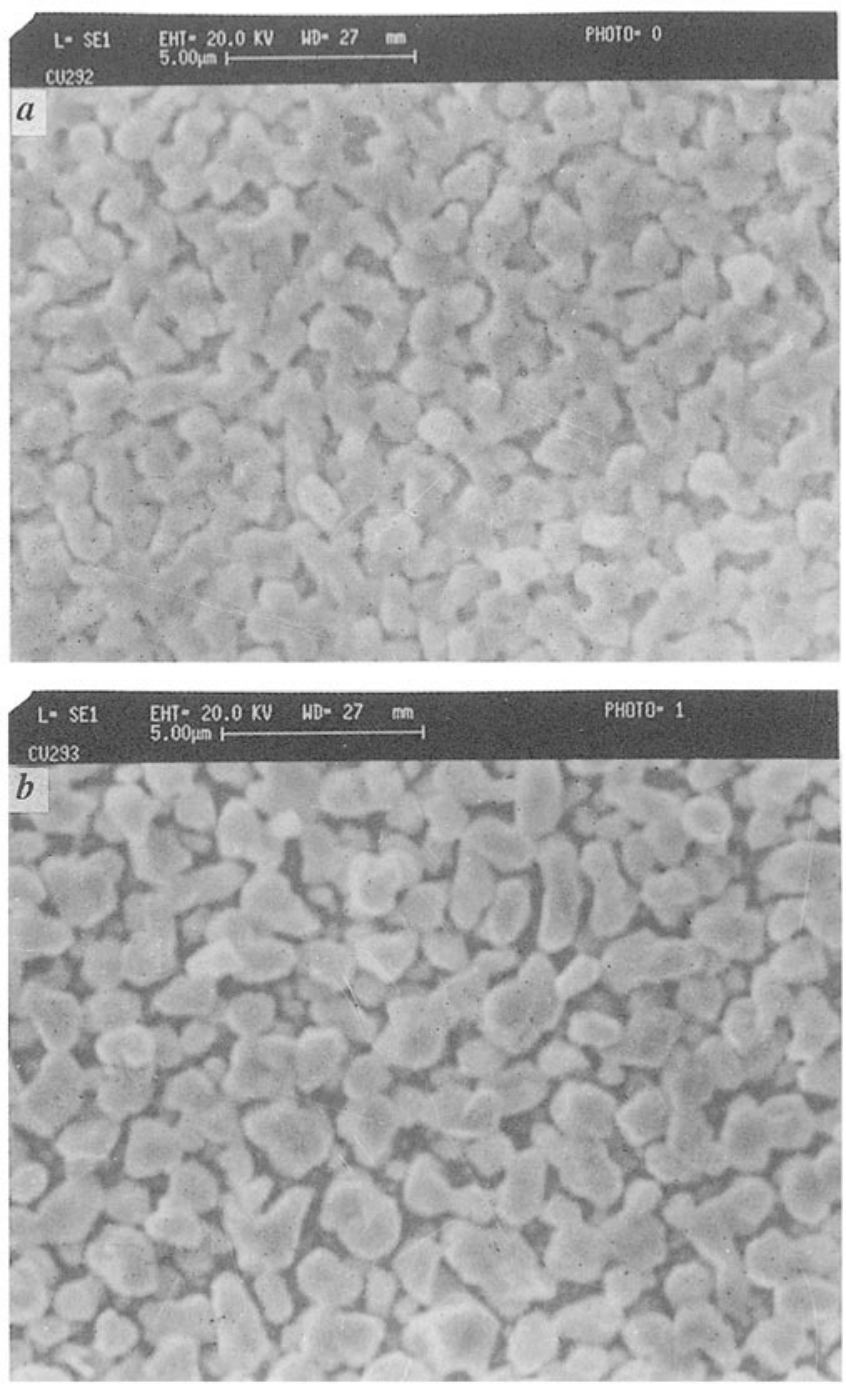

Figure 9. a-b. 

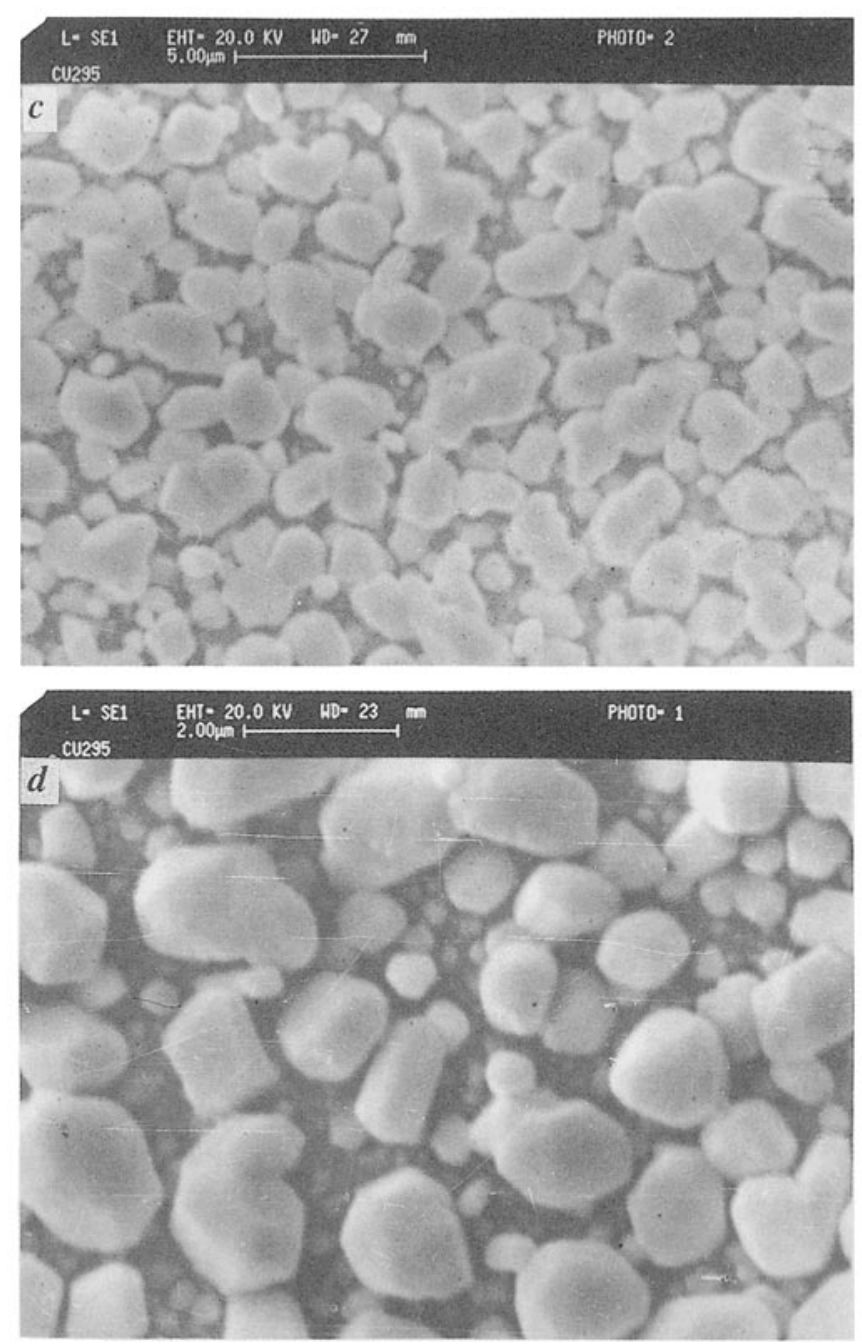

Figure 9. SEN micrograph of eopper films grown from Cu(dpm), at different substrate temperatures. (a) $350^{\circ} \mathrm{C}$. (b) $380^{\circ} \mathrm{C}$, (c) $4.50^{\circ} \mathrm{C}$ and (d) $450^{\circ} \mathrm{C}$ (higher magnification view of ' $c$ '). CVD conditions were: $P=10$ Torr and deposition timc $=45 \mathrm{~min}$.

and $E_{\mathrm{m}}^{(e)}, E_{\mathrm{m}}^{(r)}, E_{\mathrm{mil}}^{(e)}, E_{\mathrm{m}}^{(t, s)}, E_{\mathrm{m}}^{(s, a)}$, and $E_{\mathrm{vm}}^{(i, j)}$ each equal to 0.0019. The number of bursts was kept at 200 , with the size of each burst equal to 10 atoms. The total number of atoms deposited thus was held constant at 2000 for these simulations. Surface coverage was estimated by measuring the occupied sites divided by the total number of deposited atoms. When all the 2000 atoms deposited are incorporated into the first monolayer of the film, growth attains a maximum surface coverage of $80 \%$.

3.2a Case: $E_{\mathrm{aa}}<E_{\mathrm{si}}$ : In this case, with $E_{\mathrm{sa}}=1.9$ and $E_{\mathrm{a} a}=0.19$, the growth configuration (figure 14a) shows a very high surface coverage, i.e. a continuous layer 
formation is obtained with a surface coverage of $0.78 \mathrm{ML}$. Only a small fraction (2\%) of the incident atoms are involved in the formation of more than one layer. Hence the growth process appears to be layer-by-layer, where the deposited atoms attempt to complete the first layer before starting a new layer on top of it.

$3.2 \mathrm{~b}$ Case: $E_{\mathrm{uda}}=E_{\mathrm{sil}}$ : In this case, with both $E_{\mathrm{ad}}$ and $E_{\mathrm{sa}}$ taken as 1.9, the growth configuration shown in figure $14 \mathrm{~b}$ is obtained. Compared to the previous case, surface coverage is low $(0.68 \mathrm{ML})$ and a greater number of atoms $(15 \%)$ are involved in the formation of upper layers.

3.2c Case: $E_{\mathrm{a} a}>E_{\mathrm{sal}}$ : In general, film growth under this condition follows the VW
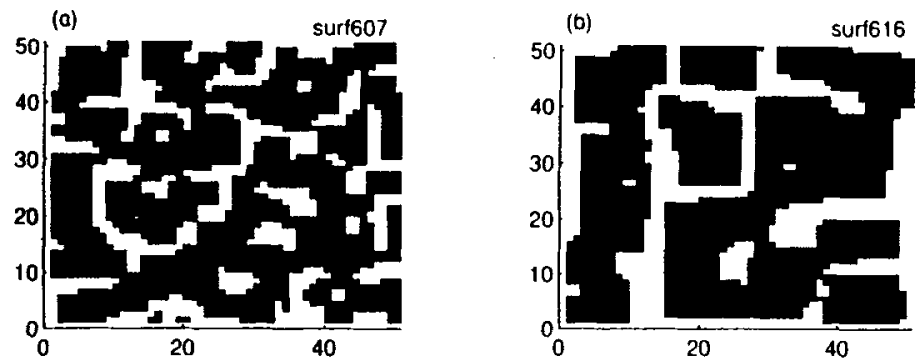

Figure 10. Growth configurations for (a) $N=100$, (b) $N=5$. The values of other parameters were: $E_{\mathrm{m}}^{(s)}=0.019, E_{\mathrm{m}}^{(\mathrm{s})}=0.0019, E_{\mathrm{in}}^{(n)}=0.0019$ and $E_{\mathrm{ill}}=1.9$ and $0.72 \mathrm{ML}$.
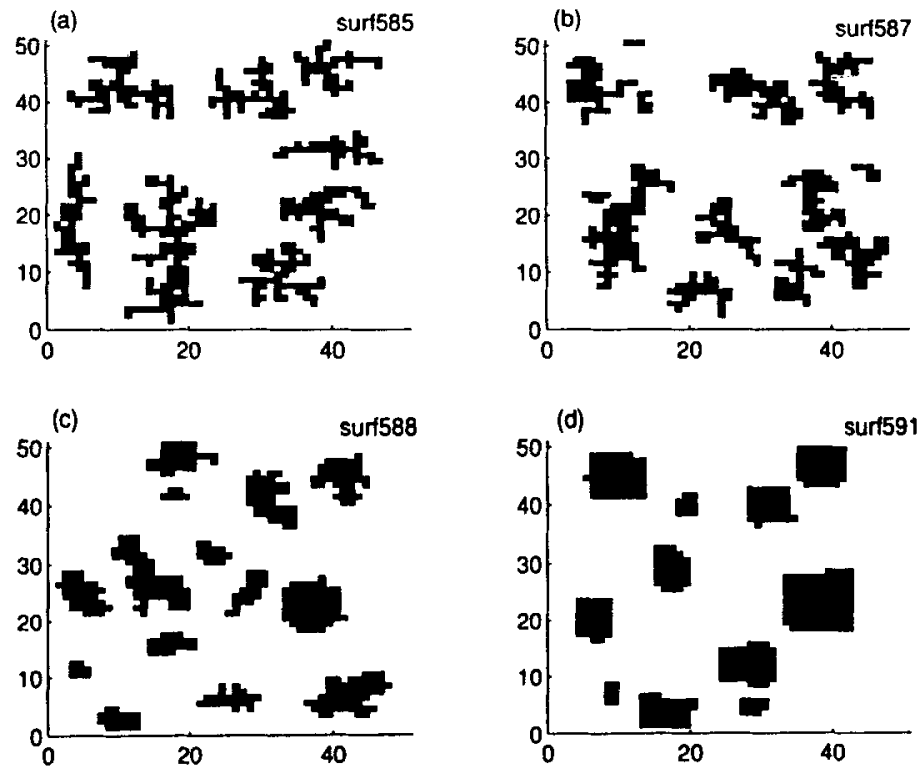

Figure 11. Growth configurations for different values of $4:$ (a) 50 , (b) 5 , (c) 1 and (d) 0.01 , 
growth mode. Simulations were carried out for two different cases. First, $E_{\mathrm{sa}}$ and $E_{\text {aa }}$ were taken as 1.9 and 190 respectively. The resulting growth configuration is presented in figure $14 \mathrm{c}$. Compared to the previous cases, the surface topography is completely different - isolated islands with more than one monolayer are formed on the substrate. In this case, surface coverage shows a low value of $0.63 \mathrm{ML}$ compared to the previous cases. In the second case, $E_{\text {sia }}$ was set at a very low value of 0.19 compared to $E_{\mathrm{da}}=190$. Figure $14 \mathrm{~d}$ shows the resulting growth configuration. Here, surface coverage is further reduced to $0.49 \mathrm{ML}$ and growth is more island-type in nature, with an increasing number of isolated clusters.

\section{Summary and conclusions}

Using a lattice-gas model in the Monte Carlo technique, computer simulations of the early stages of film growth have been carried out. The basic issue addressed

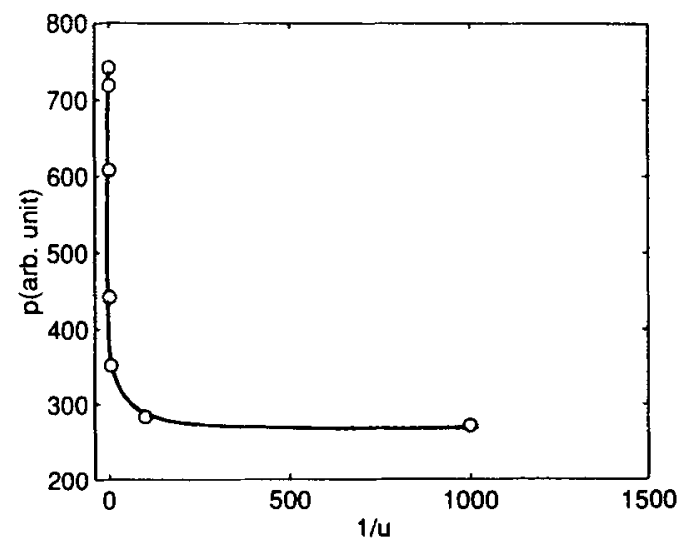

Figure 12. Plot of $p$ vs $1 / u$.
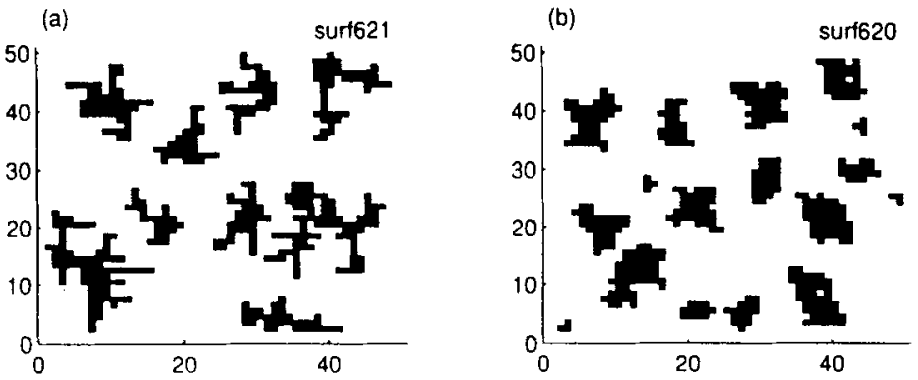

Figure 13. Growth configurations for different values of $y$ and z., (a) $y=0.01$ and $z=50$ and $(b) y=50$ and $z=0.01$. 

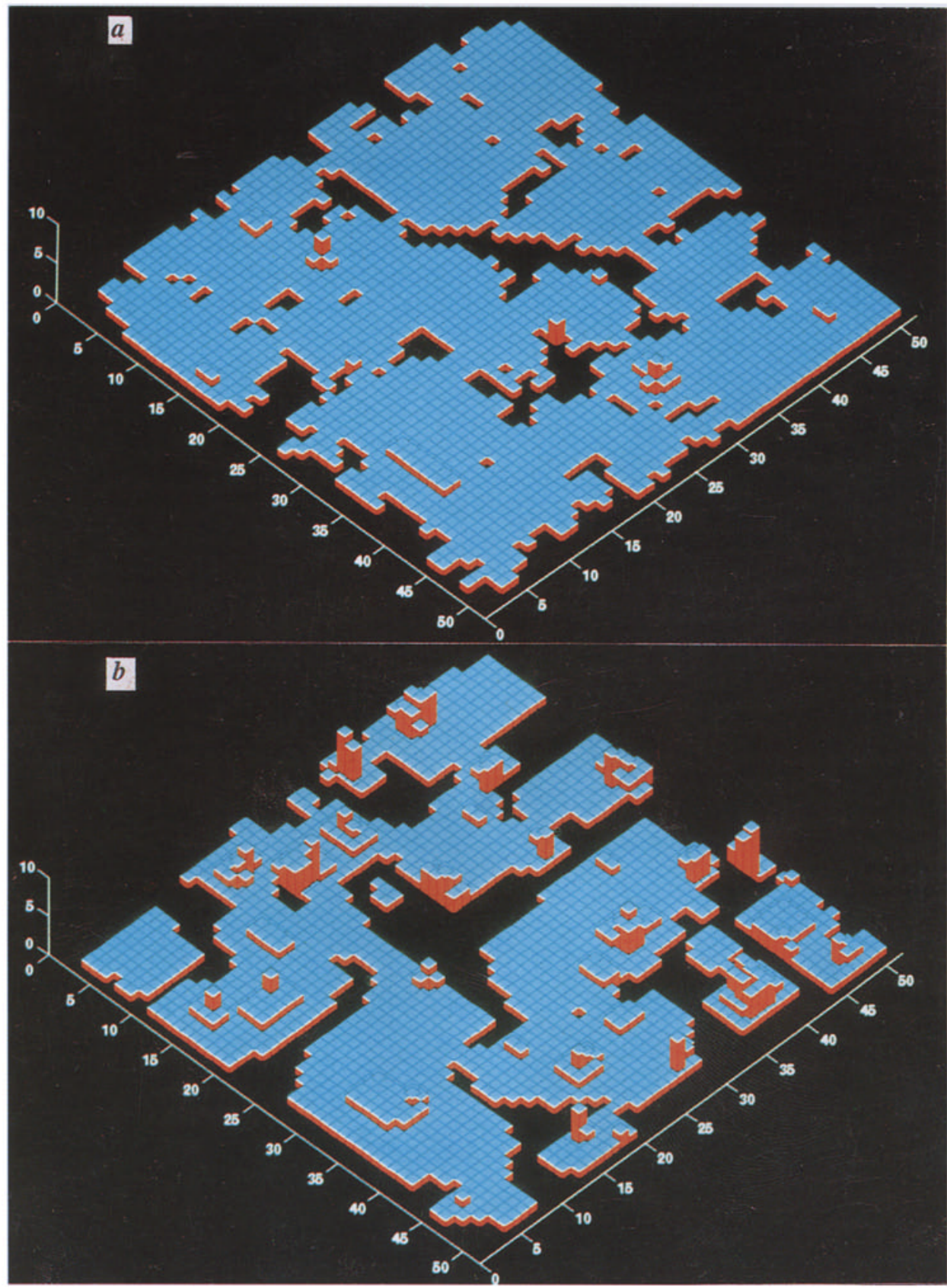

Figure 14. a-b. 


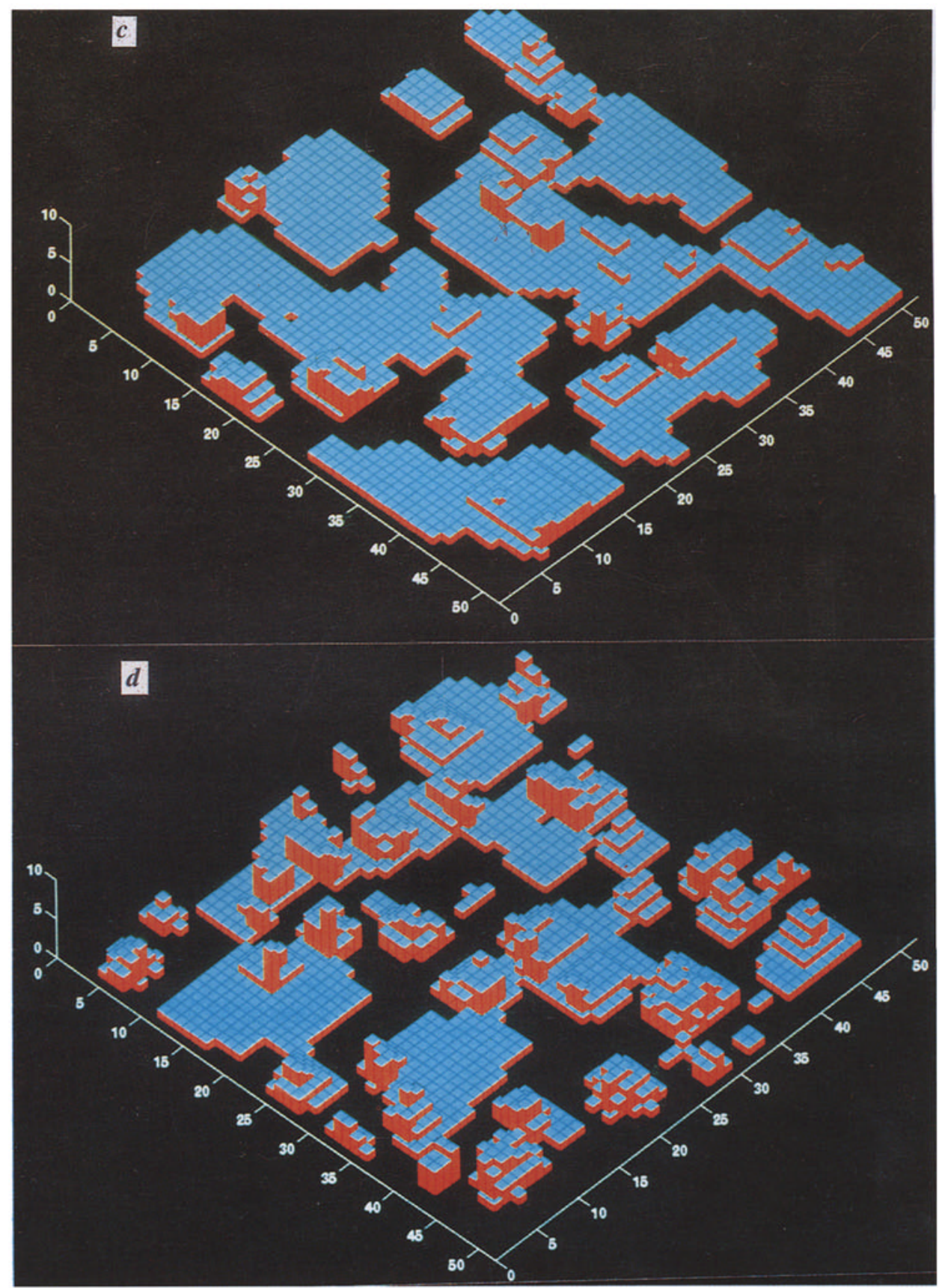

Figure 14. Growth configurations for different values of $E_{\mathrm{sia}}$ and $E_{\mathrm{iat}}:(a) E_{\mathrm{ata}}(0 \cdot 19)<E_{\mathrm{si}}$ (1.9), (b) $E_{\mathrm{ilit}}=E_{\mathrm{i}: 1}=1.9$, (c) $E_{\mathrm{itit}}(190)>E_{\mathrm{ita}}(1.9)$ and (d) $E_{\mathrm{iut}}(190) \gg E_{\mathrm{iat}}(0.19)$. 
is the effect of the relative strengths of various binding energies and migration energies on the growth morphology.

(i) With an appropriately chosen growth parameter set, this model simulates the initial stages of the film growth which is characterized by a rapid nucleation stage followed by the growth of individual clusters.

(ii) An increase of the arrival rate of the atoms increases the nucleation density, resulting in formation of surface morphology with a high density of clusters.

(iii) When surface diffusion rates are very high at a given arrival rate of the atoms, film growth approaches the minimum energy configuration with the formation of low density, large clusters with well-defined faceted shapes.

(iv) Morphological evolution as a function of surface coverage was studied for different deposition rates. A higher deposition rate for a given surface kinetics leads to the formation of well-connected microstructure at an early stage of coverage. Further, at a given deposition rate, slow surface diffusion rates result in an increase in cluster density as well as in better inter-cluster connectivity.

(v) When the capture rates of adatoms by individual clusters are faster compared to the diffusive relaxation of adatoms, governed by edge and corner diffusion, along the cluster interface, film morphology appears to attain a ramified, fractal-type structure. In contrast, a rapid diffusive relaxation leads to the formation of a compact cluster with sharp edges. At a given capture rate, the relative mobilities of adatoms, corresponding to edge and corner diffusion along the interface of the cluster, determines the shape of the clusters. While a high edge diffusion rate yields clusters with wider branches, a more compact cluster shape was observed when corner diffusion rate was very high. The best compact cluster shapes were formed when both were low.

(vi) Simulation results indicate that the relative binding energies between adatom-adatom and adatom-substrate play important roles in determining the growth mode like layer-by-layer or island-type morphology.

(vii) Even though this paper does not elaborate on the effect of dissociation (reaction) energy of incident molecular species, on the basis of results reported elsewhere, the model mimics the 'chemical effects' that influence the growth of films in CVD (Goswami et al 1997).

\section{References}

Ananthakrishna G and Noronha S J 1996 Physica A224 412

Bartelt M C and Evans J W 1994 Surf. Sci, Lett. 314 L829, L835

Brune H, Romanulnczk C, Roder H and Kern K 1994a Nature 369469

Brune H, Roder H, Boragno C and Kern K 1994b Phys. Rev. Lett. 731955

Clarke S and Vuedensky D D 1987 Phys. Rev. Lett. 723194

Clarke S and Vvedensky D D 1988 J. Appl. Phys. 632272

Edwards S F and Wilkinson D R 1982 Proc. R. Soc. London Ser. 38117

Gilmer G H, Grabow M H and Bakker A F 1990 Mater. Sci. Engg. B6 101 and the references therein

Goswami J 1995 Chemical vapour deposition of thin films of copper and $Y \mathrm{Ba}_{2} \mathrm{Cu}_{3} \mathrm{O}_{7-8}, \mathrm{Ph} \mathrm{D}$ Thesis, Indian Institute of Science, Bangalore

Goswami J, Raghunathan L, Devi A, Shivashankar S A and Chandrasekaran S 1996 J. Mater. Sci. Lett. 15573

Goswami J, Shivashankar S A and Ananthakrishna G 1997 Thin Solid Films (to appear) 
Hwang R Q, Schroder J, Gunther C and Behm R J 1991 Phys. Rev. Lett. 673279

Kaneko T, Kageyama K, Sasaki T and Yamamoto R 1989 Jph. J. Appl. Phys. 281900

Lai Z W and Das Sarma S 1991 Phys. Rev. Lett. 662348

Michely T, Hohage M, Batt M and Cosma G 1993 Phys. Rev. Lett. 703943

Mo Y W, Kleiner J, Webb M B and Lagally M G 1991 Phys. Rev. Lett. 661998

Ratch C, Zangwill A. Smilauer P and Vvedensky D D 1994 Phys. Rev. Lett. 723194

Roder H, Hahn E, Brune H, Bucher J P and Kern K 1993 Nature 366141

Roder H, Bromann K, Brune H and Kern K 1995 Phys. Rev. Lett. 743217

Venables J A $1986 \mathrm{~J}$. Vac: Sri. Technol. B4 870

Venables J A, Spiller G D T and Hanbucken M 1984 Rep. Prog. Phys. 47399

Villain J 1991 J. Phys. I 119

Witten T A and Sander L M 1981 Phys. Rev. Lett. 471400

Xu Shi and Lu G Q 1994 J. Mater. Sci. Lett. 131629

Zhang Z, Chen X and Lagally M G 1994 Phys. Rev. Lett. 731829 